\title{
Fetal Internal Carotid and Umbilical Artery Blood Flow Velocity Waveforms as a Measure of Fetal Well-Being in Intrauterine Growth Retardation
}

\author{
J. W. WLADIMIROFF, M. J. NOORDAM, J. A. G. W. VAN DEN WIJNGAARD, AND W. C. J. HOP \\ Department of Obstetrics and Gynaecology [J.W.W., M.J.N., J.A.G.W.v.d.W.] and Department of Biostatistics \\ [W.C.J.H.], Erasmus University Rotterdam, Rotterdam, The Netherlands
}

\begin{abstract}
Maximal flow velocity waveforms were recorded on one occasion from the umbilical artery (UA) and fetal internal carotid artery (ICA) in 240 normal pregnancies and 44 cases of intrauterine growth retardation between 26 and 39 wk of gestation. In normal pregnancy the mean UA pulsatility index (PI) decreased from 1.14 (SD: \pm 0.13 ) at $26-27$ wk of gestation to 0.78 (SD: \pm 0.15$)$ at 38-39 wk. The corresponding decrease in ICA PI was from 1.63 (SD: \pm 0.19 ) to 1.31 (SD: \pm 0.21 ). Mean values of UA PI for normal pregnancies were linearly related to gestational age; for ICA PI this relation appeared to be quadratic. Normal limits according to age were constructed by estimated means $\pm 2 \mathrm{SD}$. In intrauterine growth retardation, the UA PI was increased ( $>2$ SD) in $80 \%$ of cases, ICA PI was reduced ( $>2 \mathrm{SD}$ ) in only $45 \%$. The outcome of fetuses with intrauterine growth retardation, as expressed by fetal heart rate abnormality, Apgar score at 1 min, and umbilical cord $\mathrm{pH}$, was significantly related to the UA PI but not to the ICA PI. (Pediatr Res 24: 609612,1988 )
\end{abstract}

\section{Abbreviations}

FHR, fetal heart rate

ICA, internal carotid artery

IUGR, intrauterine growth retardation

PI, pulsatility index

UA, umbilical artery

Recently, a Doppler ultrasound method for recording the blood flow velocity waveform in the fetal internal carotid artery was described (1).

Preliminary results suggested that in IUGR due to impaired placental perfusion there was a higher percentage of abnormal PI values in the UA than in the ICA (2). It was also suggested that the reduction in ICA PI reflects redistribution of blood flow in the fetus with the aim of maintaining optimal oxygen supply to the fetal brain (2). The question arises as to if this redistribution of blood flow reflects a further deterioration in fetal oxygen supply in the presence of IUGR.

The objective of the present study was 3-fold: 1) to establish the normal distribution of UA PI and ICA PI during the third trimester of pregnancy; 2 ) to determine the degree of abnormality

Received March 21, 1988; accepted July 6, 1988. Correspondence J. W. Wladimiroff, M.D., Ph.D., Professor of Obstetrics and Gynaecology, Academic Hospital Rotterdam-Dijkzigt, Erasmus University Rotterdam, Dr. Molewaterplein 40, 3015 GD Rotterdam, The Netherlands. of UA PI and ICA PI during this period of gestation in the presence of IUGR due to impaired placental perfusion; 3) to relate UA PI and ICA PI to fetal well-being as expressed by antenatal fetal heart rate patterns before delivery, Apgar score at $1 \mathrm{~min}$, and umbilical arterial $\mathrm{pH}$.

\section{SUBJECTS AND METHODS}

A combined mechanical sector and pulsed Doppler system (Diasonics CV 400) with a carrier frequency of 3.5 and $3 \mathrm{MHz}$ was used for blood flow velocity measurements in the fetal internal carotid artery and UA. The energy output of the pulsed Doppler transducer was $1.5 \mathrm{~mW} / \mathrm{cm}^{2}$ (spatial peak, temporal average). The maximum flow velocity waveform in the internal carotid artery was obtained at the level of the bifurcation into the middle and anterior cerebral artery (1). The sample size of the Doppler probe, necessary for sampling frequency shifts originating from the moving erythrocytes within a vessel, was $4 \mathrm{~mm}$. This allowed clear signals from the internal carotid artery without interference from other nearby vessels such as the middle and anterior cerebral artery. The maximum flow velocity waveform in the UA was recorded using the method first described by McCallum et al. (3). All flow velocity waveform records were obtained during fetal apnea to avoid modulation of the waveforms by fetal breathing movements. Therefore, only waveforms without visible changes in the waveform pattern were accepted. The degree of pulsatility of the waveform was quantified by calculating the PI according to the following formula (4): fmax - fmin/fmean, in which fmax is the maximum Doppler frequency shift, fmin is the minimum Doppler frequency shift, and fmean is the mean Doppler frequency shift over the entire cardiac cycle. In both flow velocity waveforms, the PI was calculated by

Table 1. Means and SD of UA PI and ICA PI according to duration of gestation, grouped in 2-wk intervals for cases with normal pregnancies

\begin{tabular}{cccccccc}
\hline \multirow{2}{*}{$\begin{array}{c}\text { Gestational age } \\
(\mathrm{wk})\end{array}$} & \multicolumn{3}{c}{ UA PI } & & \multicolumn{3}{c}{ ICA PI } \\
\cline { 2 - 4 } \cline { 6 - 8 } & Mean & SD & $n$ & & Mean & SD & $n$ \\
\hline $26-27$ & 1.14 & 0.13 & 17 & & 1.63 & 0.19 & 14 \\
$28-29$ & 1.06 & 0.14 & 23 & & 1.58 & 0.15 & 16 \\
$30-31$ & 1.05 & 0.18 & 32 & & 1.55 & 0.20 & 24 \\
$32-33$ & 1.00 & 0.15 & 32 & & 1.61 & 0.21 & 27 \\
$34-35$ & 0.89 & 0.14 & 29 & & 1.58 & 0.21 & 33 \\
$36-37$ & 0.82 & 0.14 & 50 & & 1.37 & 0.27 & 50 \\
$38-39$ & 0.78 & 0.15 & 42 & & 1.31 & 0.21 & 41 \\
\hline
\end{tabular}


a microcomputer. The mean value over at least four cardiac cycles represented the PI for a particular pregnancy.

A total of 240 normal pregnancies and 44 cases of IUGR participated in the study after oral informed consent was obtained. Gestational age varied between 26 and 39 wk. Each subject was certain of the onset of the last menstrual period.

Normal pregnancy was defined by a normal biparietal diameter on ultrasound and a birth weight between the 10th and 90th percentile for gestational age, corrected for maternal parity and fetal sex (5). Each subject was included in the study only once. The median maternal age was $27 \mathrm{yr}$ (range 17-41 yr), the median parity was 2 (range $1-6$ ).

Acceptable flow velocity waveforms for PI calculations were obtained from the ICA in $205(85 \%)$ and from the UA in 225 ( $94 \%)$ of the 240 normal subjects. IUGR was defined by: 1 ) flattening of the growth pattern resulting in a clinical discrepancy of more than $2 \mathrm{wk}$ on fundal height on two successive appointments combined with an ultrasound finding of the upper abdom-
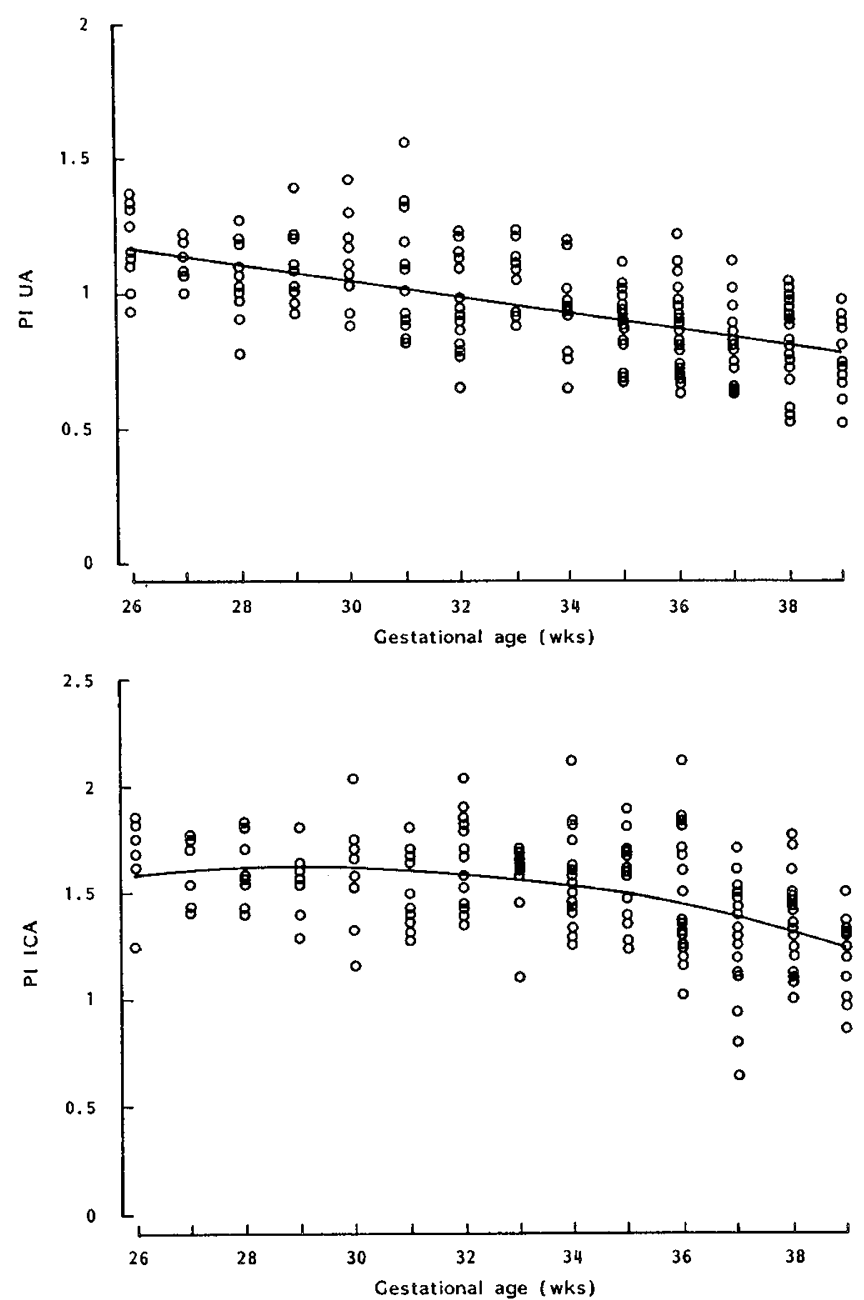

Fig. 1. UA PI and ICA PI of normal subjects. Least squares fitted curves are: $\mathrm{UA} \mathrm{PI}=1.99-0.032^{(1)} \times$ age and ICA PI $=-1.69+0.23^{(2)}$ $\times$ age $-0.004^{(3)} \times$ age square. SE of coefficients: 0.003 for (1), 0.08 for (2) and 0.001 for (3). inal circumference measurement below the 10th percentile in association with a normal or reduced head circumference measurement (6) and 2) postnatal confirmation by a birth weight below the 10th percentile for gestational age, corrected for maternal parity and fetal sex (5). The lagtime between flow velocity records and delivery in the 44 cases of IUGR ranged between 2 $\mathrm{h}$ and 14 days (median 4 days). Occasionally more than one measurement was done per patient. In such cases the measurement nearest to the date of delivery was chosen for analysis. Median maternal age was 28 yr (range $18-40 \mathrm{yr}$ ), median parity 2 (range 1-5). In 38 subjects PI values from both vessels were related to the Apgar score at $1 \mathrm{~min}$; in 25 subjects also to UA $\mathrm{pH}$. In 35 cases PI values from both vessels were related to FHR patterns before the onset of labor or elective cesarean section.
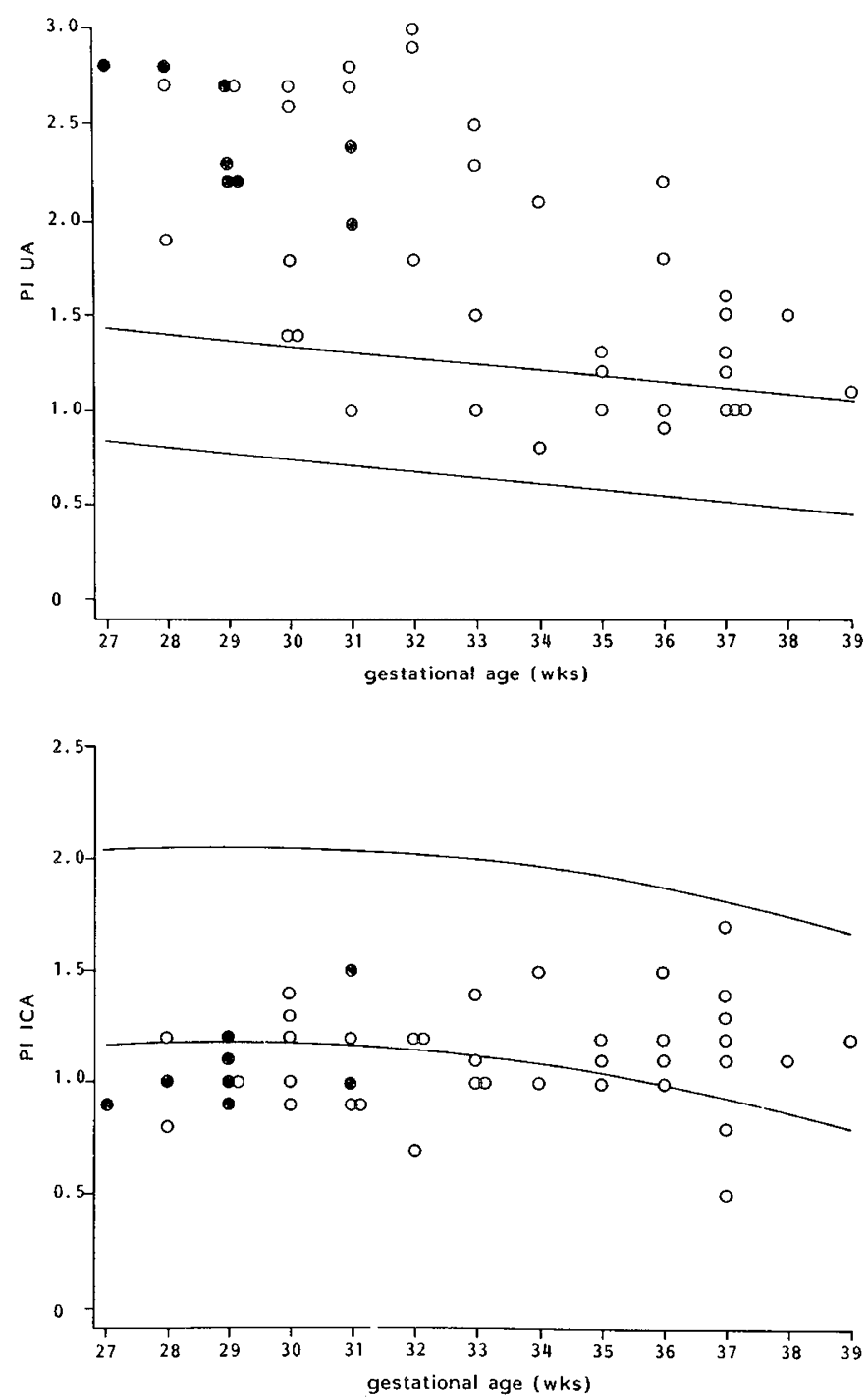

Fig. 2. UA PI and ICA PI for IUGR cases $(n=44)$ according to gestational age. Plotted curves represent normal limits (means \pm 2 SD). Closed circles denote perinatal deaths.

Table 2. Numbers of IUGR cases according to gestational age, UA PI, and ICA PI

\begin{tabular}{|c|c|c|c|c|c|c|}
\hline \multirow{2}{*}{$\frac{\text { Gestational age }}{\text { ICA PI }}$} & \multicolumn{3}{|c|}{$27-32 \mathrm{wk}$} & \multicolumn{3}{|c|}{$33-39$ wk } \\
\hline & Decreased & Normal & Total & Decreased & Normal & Total \\
\hline Increased UA PI & 13 & 8 & $21(95 \%)^{*}$ & 6 & 8 & $14(64 \%) \dagger$ \\
\hline Normal UA PI & 0 & 1 & 1 & 1 & 7 & 8 \\
\hline Column total & $13(62 \%)^{*}$ & 9 & 22 & $7(32 \%) \dagger$ & 15 & 22 \\
\hline
\end{tabular}

\footnotetext{
Significantly different: ${ }^{*} p=0.008, \dagger p=0.04$.
} 

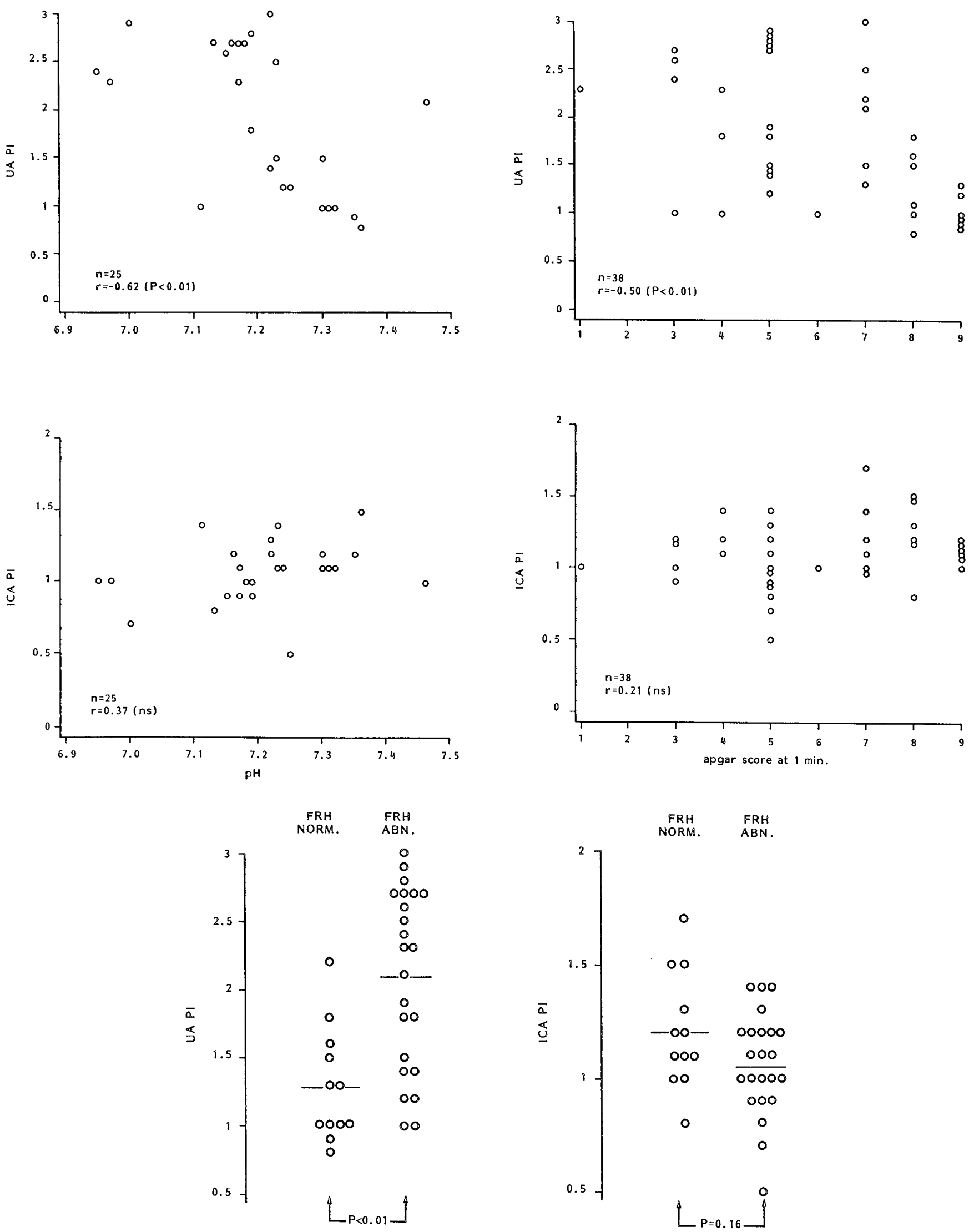

Fig. 3-5. UA PI and ICA PI versus UA pH (Fig. 3), Apgarscore at 1 min (Fig. 4) and FHR (Fig. 5) during IUGR.

FHR was recorded using a Doppler ultrasound cardiotocograph (Hewlett Packard 8040A, carrier frequency $1 \mathrm{MHz}$ ). A normal FHR pattern was defined as a pattern with a baseline varying between $110-160$ beats/min, with good beat to beat variability, periodic accelerations, and no decelerations. An abnormal FHR pattern was one that deviated from this normal definition.

Statistical analysis included investigation of the relations between the pulsatility indices and gestational age by polynomial 
regression. Differences in the degree of abnormality of UA PI and ICA PI in IUGR were tested by McNemars test. Differences in distributions of PI were tested by use of the Mann-Whitney test in comparing two groups. Correlation coefficients given are according to Spearman.

\section{RESULTS}

Table 1 shows the mean PI of both arteries as a function of gestational age, grouped in 2-wk intervals. The mean of each index varied with gestational age, whereas the SD did not differ significantly between the age groups.

By polynomial regression, it appeared that the mean UA PI could be described by a linear function of gestational age (Fig. 1, upper panel). No significant improvement of the curve's fit could be obtained by adding quadratic or cubic components of gestational age.

For the internal carotid artery, the mean PI (ICA PI) could be described by a quadratic function of gestational age (Fig. 1, lower panel). Addition of a cubic component of gestational age did not significantly improve the fit of the curve. The SD of the measurements around the fitted curves for the UA PI and the ICA PI were 0.15 and 0.20 , respectively. In establishing normal limits according to gestational age, these SD were used by taking estimated means $\pm 2 \mathrm{SD}$.

In Figure 2, these limits are graphed for both PI. Also in Figure 2 , the data points are plotted for the 44 cases with IUGR. For the UA PI, the majority of IUGR cases $(80 \%)$ showed increased $(>2 \mathrm{SD})$ values. For the ICA PI, $45 \%$ of IUGR cases showed decreased $(>2 \mathrm{SD})$ measurements.

The percentage of cases of IUGR with an increased UA PI was significantly greater than the percentage with a decreased ICA PI. This applied to the shorter gestational ages as well as to the longer ones (Table 2).

It appears that the measured values of UA PI in IUGR pregnancies at longer gestational ages were generally lower than at shorter ages $(r=-0.64 ; p<0.001)$ and approached normal values with increasing gestational age. The mean UA PI [1.23 \pm 0.25 (SD)] of the seven IUGR cases studied at wk 37 was, however, still significantly greater $(p<0.001)$ than the mean index $[0.81 \pm 0.14(\mathrm{SD})]$ of the 19 normal subjects studied at the same age.

With respect to the ICA PI no significant correlation with gestational age was present in the IUGR cases $(r=0.25 ; p>$ $0.10)$. The mean value of the ICA PI at $37 \mathrm{wk}[1.14 \pm 0.40$ (SD)] for IUGR cases did not differ significantly from the mean [1.30 \pm 0.26 (SD)] of the normal subjects studied at the same age.

In Figures 3 to 5 , the fetal well-being of the IUGR cases, as reflected by the umbilical arterial $\mathrm{pH}$ (Fig. 3), Apgar score at 1 min (Fig. 4) and FHR pattern (Fig. 5) is related to UA PI and ICA PI. All three indicators correlated significantly with UA PI. The mean UA PI in cases with abnormal FHR was significantly higher than at normal FHR. No correlation could be established between the three indicators of fetal well-being and ICA PI. There were eight perinatal deaths, five of which occurred in utero and three within 7 days after delivery. All deaths were associated with increased PI values in the UA and five cases were associated with reversed end-diastolic flow. ICA PI was reduced in six of the eight cases.

\section{DISCUSSION}

The potential clinical use of flow velocity waveform records from the fetal ICA is demonstrated by the $85 \%$ success rate in obtaining such records. Failure to demonstrate the fetal ICA was usually due to excessive fetal movements or maternal obesity.
The PI from the UA shows a significant decrease during the third trimester of pregnancy, reflecting a reduction in placental vascular resistance, as has been established by other studies $(7-10)$. The PI from the fetal ICA shows a fairly constant pattern up to 32-33 wk of gestation followed by a decrease toward term. Inasmuch as a gradual reduction in fetal $\mathrm{pO}_{2}$ during the last 45 wk of gestation has been observed (11), this decrease in PI may reflect a relative increase in fetal internal carotid blood flow to maintain cerebral oxygenation. The decrease in PI from the fetal ICA during IUGR is mainly due to changes in end-diastolic flow velocity and confirms earlier data suggesting a redistribution of blood flow favoring oxygen supply to the fetal brain in growthretarded fetuses (2).

Our study indicates that in IUGR due to impaired placental perfusion, a considerable percentage $(55 \%)$ of ICA PI values is within the normal range. We suggest that, in the presence of a raised PI from the UA, a normal PI from the ICA may already reflect some kind of redistribution of blood flow. Fetal compromise, as expressed by an abnormal FHR tracing, low umbilical arterial $\mathrm{pH}$, and low Apgar score, correlated well with UA PI, suggesting that this flow variable may be helpful in recognizing fetal compromise. Support for this can be found in a recent comparative study between UA flow velocity waveform and antenatal fetal heart rate monitoring (12). Fetal compromise was more efficiently recognized by the UA waveforms, with two to three times higher sensitivity and similar positive and negative predictive values. Even more important was the identification of those fetuses at risk of further morbidity, i.e. admission to neonatal intensive care.

In our study, no correlation could be documented between the indicators of fetal well-being and ICA PI. Whereas a reduction in ICA PI may reflect blood flow redistribution as a result of chronic fetal hypoxia, the degree of reduction of this variable does not seem to accurately reflect fetal welfare.

It can be concluded that flow velocity measurements in the $\mathrm{UA}$ are helpful in predicting fetal well-being, whereas this is not true for flow velocity measurements in the ICA.

\section{REFERENCES}

1. Wladimiroff JW, Tonge HM, Stewart PA 1986 Doppler ultrasound assessment of cerebral blood flow in the human fetus. Br J Obstet Gynaecol 93: 471475

2. Wladimiroff JW, Wijngaard van den JAGW, Degani S, Noordam MJ, Eyck van J, Tonge HM 1987 Cerebral and umbilical arterial blood flow velocity waveforms in normal and growth retarded pregnancies; a comparative study. Obstet Gynaecol 69:705-709

3. MacCallum WD, Williams CS, Napel S, Daigle RE 1978 Fetal blood velocity waveforms. Am J Obstet Gynecol 132:425-429

4. Gosling RG, King DH 1975 Ultrasound angiology. In: Marcus AW and Adamson L (eds) Arteries and Veins. Churchill Livingstone, Edinburgh, pp 61-98

5. Kloosterman GJ 1970 On intrauterine growth. Int J Gynaecol Obstet 8:895912

6. Campbell S 1976 Fetal growth. In: Beard RW, Nathanielsz PW (eds) Fetal Physiology and Medicine. WB Saunders \& Co. Ltd., London, pp 271-279

7. Reuwer PJHM, Bruinse HW, Stoutenbeek PH, Haspels AA 1984 Doppler assessment of the fetoplacental circulation in normal and growth retarded fetuses. Eur J Obstet Gynecol Reprod Biol 18:199-207

8. Trudinger BJ, Giles WB, Cook CM 1985 Flow velocity waveforms in the maternal uteroplacental and fetal umbilical placental circulations. Am J Obstet Gynecol 152:155-160

9. Stuart B, Drumm J, FitzGerald DE, Duignan NM 1980 Fetal blood velocity waveforms in normal pregnancy. Br J Obstet Gynaecol 87:780-785

10. Fleisher A, Schulman H, Farmakides G, Bracero L, Blattner P, Randolph G 1985 Umbilical artery velocity waveforms and intrauterine growth retardation. Am J Obstet Gynecol 151:502-505

11. Soothill, PW, Nicolaides KH, Rodeck CH, Campbell S 1986 Effect of gestational age on fetal and intervillous bloodgas and acid-base values in human pregnancy. Fetal Therapy 4:168-175

12. Trudinger BJ, Cook CM, Jones L 1986 A comparison of fetal heart rate monitoring and umbilical artery waveforms in the recognition of fetal compromise. Br J Obstet Gynaecol 93:171-175 